\title{
Menstruating umbilicus! A rare case report of primary umbilical endometriosis with review of literature
}

\author{
Renuka Malik*, Preeti Sharma
}

Department of Obstetrics and Gynaecology, PGIMER, RML Hospital, New Delhi, India

Received: 06 May 2016

Revised: 03 June 2016

Accepted: 04 June 2016

\section{*Correspondence:}

Dr. Renuka Malik,

E-mail: renucam@yahoo.co.in

Copyright: () the author(s), publisher and licensee Medip Academy. This is an open-access article distributed under the terms of the Creative Commons Attribution Non-Commercial License, which permits unrestricted non-commercial use, distribution, and reproduction in any medium, provided the original work is properly cited.

\begin{abstract}
Umbilical endometriosis (UE) represents $0.5 \%$ to $1 \%$ of all cases of extra genital endometriosis. Primary umbilical endometriosis is the presence of ectopic endometrial tissue located in the umbilicus in absence of previous surgery for either gynecological disorders or cesarean section which is defined as secondary umbilical endometriosis. Secondary UE, which is more common than primary UE and is probably due to the iatrogenic dissemination and implant of endometrial cells during either laparoscopic or open surgical procedures. Mrs. X, presented in OPD with an umbilical nodule since 6 months which grew in size and became painful and bled during periods. Imaging studies were done including MRI. A provisional diagnosis of umbilical endometriosis was made as patient had no history of any prior surgery. Patient had no suggestive history of pelvic endometriosis. Diagnostic laparoscopy was done to rule out concomitant pelvic endometriosis followed by wide local excision and umbilical reconstruction was done. By plastic surgeons. Knowledge of this rare condition and correct management is important to avoid recurrence. Medical treatment is only palliative not definitive in this condition.
\end{abstract}

Keywords: Umbilical nodule, Extra pelvic endometriosis, Primary umblical endometriosis

\section{INTRODUCTION}

Extra pelvic endometriosis accounts up to $15 \%$ of all cases of endometriosis. Umbilical endometriosis has been reported to be around $0.4-4 \%$ of all patients with endometriosis and accounts for up to $30-40 \%$ of all cases of cutaneous endometriosis. ${ }^{1}$ Other more common manifestation then primary umbilical endometriosis is scar endometriosis occurring in laparotomy scar. Primary umbilical endometriosis is a rare presentation, entity known among gynaecologists, but possibly unfamiliar to many other specialists. Due to the rarity of this entity, no guidelines for treatment exist. We report this case for its rarity with review of literature from 1990 onwards for optimal management of this condition. Written consent obtained from patient for publication of case and images.

\section{CASE REPORT}

Mrs. X, 45 year woman, para 2, presented to gynae OPD of DR. RML hospital, New Delhi on 6 February 2016, with complaints of pain and cyclical bleeding from her umbilicus for last 6 months. She also gave history of an umbilical nodule, which she first noticed 11 years back which was asymptomatic but since last 6 months had increased in size and had become painful. She gave history of increase in size and bleeding from nodule since last 5 months during her periods. There was no previous history of any surgery. There was no other history of pain abdomen, any menstrual irregularity, or bowel or urinary complaints. On examination a small 0.8 x $0.8 \mathrm{~cm}$ tender nodule was seen in umbilicus [Figure 1(A)]. P/S and P/V examination revealed 2 degree cervical descent with normal size uterus, mobile with no tenderness or any mass present in any fornix. To confirm her complaint, 
patient was asked to come during periods. When she reported in OPD during periods, the nodule had increased in size and blood was seen coming out of umbilicus [Figure 1 (B)]. A provisional diagnosis of primary umbilical endometriosis was made and patient was further investigated. MRI was done which revealed localized infiltration below umbilicus [Figure $1(\mathrm{C})$ ] and no other evidence of endometriosis in pelvis. Patient was scheduled for wide local excision of nodule with diagnostic laparoscopy in premenstrual phase to exclude pelvic endometriosis and also umbilical reconstruction by plastic surgeons.

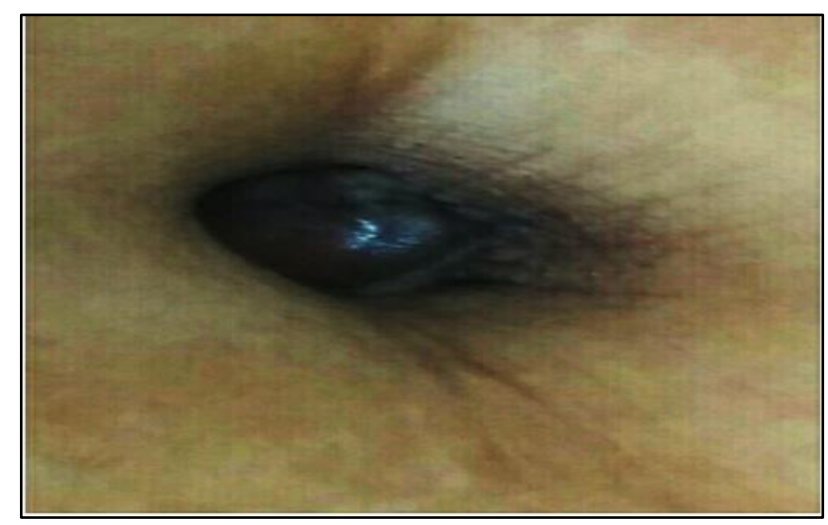

Figure 1(A): Umbilical nodule.

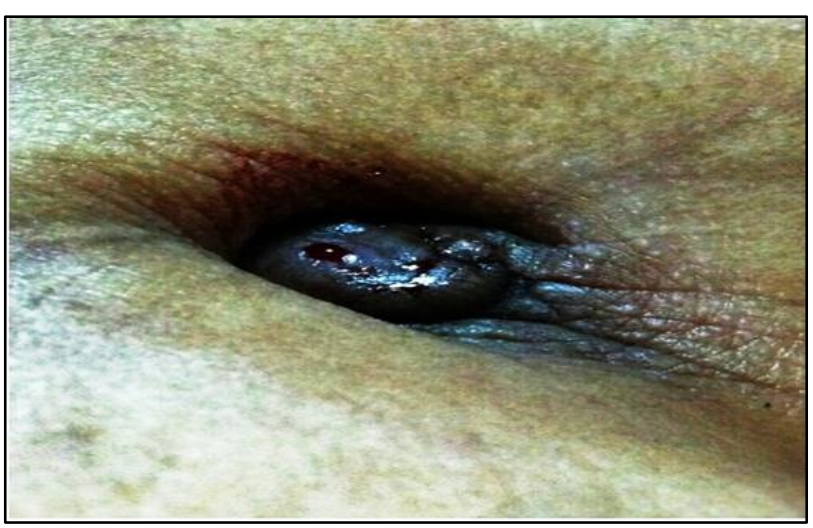

Figure 1(B): Menstruating umbilicus.

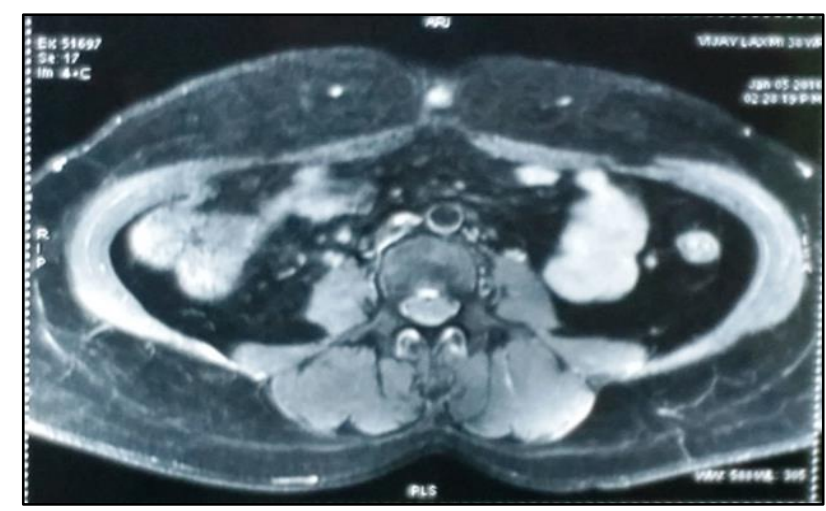

Figure 1(C): MRI hyper intense lesion suggestive of umbilical endometriosis.

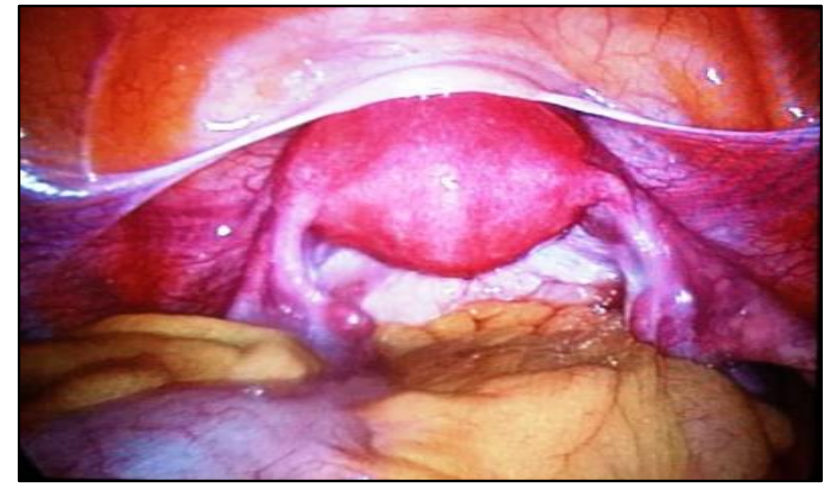

Figure 2(A): No evidence of pelvic endometriosis on laparoscopy.

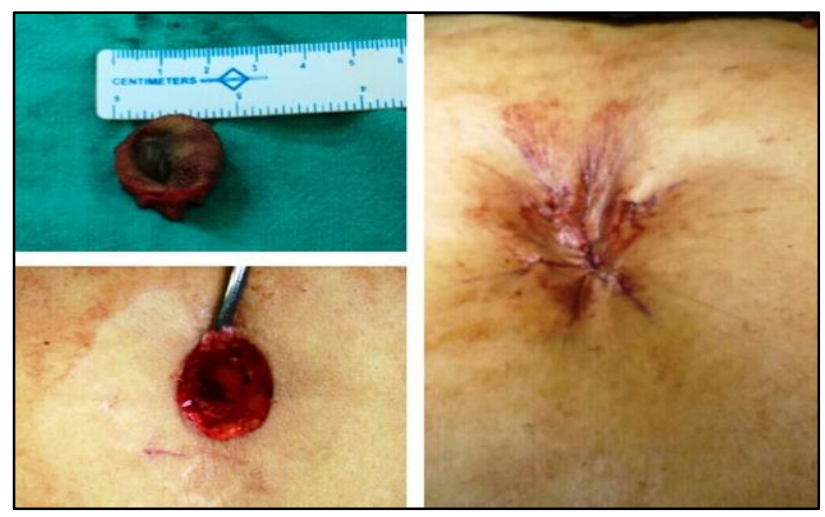

Figure 2(B): Excised umbilicus with endometriosis and reconstructed umbilicus.

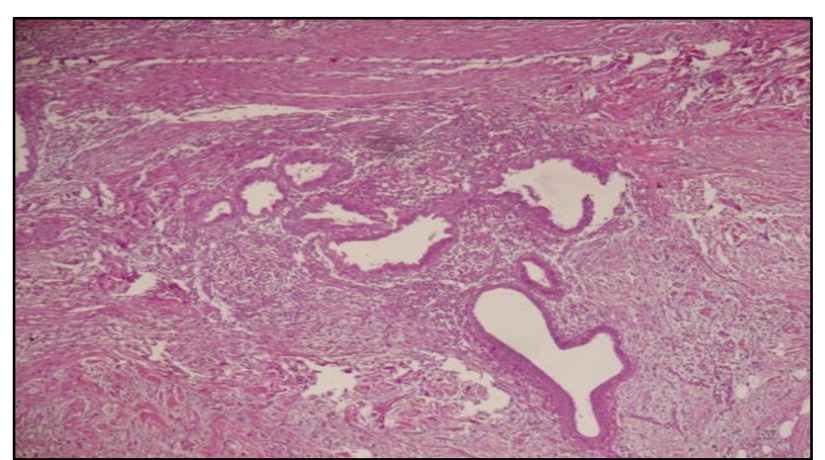

Figure 2(C): Histopathology presence of endometrial glands and stroma in subcutaneous tissue and rectus sheath.

Supraumbilical insertion of verees needle was done and $10 \mathrm{~mm}$ port put supraumbilically, well avoiding the nodule. Diagnostic laparoscopy revealed normal uterus and ovaries with no evidence of endometriosis in pelvis [Figure 2 (A)]. There appeared no extension below umbilicus. A wide local excision was done, extending the supraumbilical incision all around nodule [Figure 2 (B)]. Umbilicus was reconstructed by plastic surgeons. Histopathology confirmed the presence of endometrial glands in the umbilicus with free margins [(Figure 2 (C)]. As there was no sign of endometriosis at any other site, 
patient after wide local excision of umbilical nodule was not put on any other medical therapy in follow-up. Patient remains in follow up with complete relief of her symptoms.

\section{DISCUSSION}

Endometriosis, a term first used by Sampson, is the presence of endometrial glands and stroma outside the uterine cavity and musculature. It affects $7 \%$ to $10 \%$ of women in the reproductive age group. It commonly occurs in the pelvic organs, especially the ovaries, the utero sacral ligaments and the pouch of Douglas. Women with endometriosis often present with dysmenorrhea, $\mathrm{HMB}$, pelvic pain, intestinal and urinary complaints and infertility.

Extra genital endometriosis is less common, but has been described in almost every area of the female body including the bowel, bladder, lungs, brain, umbilicus, and surgical scars even in male's prostate and bladder. Due to its varied presentations, endometriosis remains a difficult condition to diagnose and treat. Umbilical endometriosis (UE), also known as Villar's nodule from the first physician describing the disease, is a very rare entity. It was first described by Villar in 1886 . UE represents $0.5 \%$ to $1 \%$ of all cases of extra genital endometriosis. Primary umbilical endometriosis is the presence of ectopic endometrial tissue located in the umbilicus in absence of previous surgery. UE following any surgery caesarean, laparotomy, or laparoscopy is defined as secondary umbilical endometriosis. Secondary UE, which is more common than primary UE and is probably due to the iatrogenic dissemination and implant of endometrial cells during either laparoscopic or open surgical procedures.

A review of literature of primary umbilical endometriosis was done, in English language from 1990 to 2016, using pub med data base Search engine. 64 studies have been published since 1990. 74 cases have been reported in these studies and presentation with treatment compared. This is the $75^{\text {th }}$ reported case. Surprisingly, only in 28.76 (20) $\%$ of patients was pelvic endometriosis. Associated as in rest, as seen in our case there was no evidence of pelvic endometriosis. Mean duration of symptoms was 18 months and mean size was $20 \mathrm{~mm}$. In our case the duration of symptoms was 6 months. The diagnosis was made early as patient reported to gynecologists, who are well versed with endometriosis.

Different hypothesis have been proposed to understand the origin of endometriosis Latcher has classified these theories into three main categories: the embryonal rest theory, which explains endometriosis adjoining the pelvic viscera by Wollfian or Mullerian remnants; the coelomic metaplasia theory, which states that the embryonic coelomic mesothelium dedifferentiates into endometrial tissue under stimulus such as inflammation or trauma and the migratory pathogenesis theory, which explains the dispersion of endometrial tissue by direct extension, vascular and lymphatic channels and surgical manipulation. ${ }^{2}$ Still others suggest cellular proliferation of endometrial cells from initial extra peritoneal disease along the urachus. As described by Mizutani et al who found urachus attached to base of umbilical endometriotic nodule. ${ }^{3}$ However, the pathogenesis of primary endometriosis still remains unclear.

The coelomic metaplasia theory, the metaplastic transformation into endometrial cells would explain the exceptional formation of foci in the bladder and prostate of males. In primary umbilical endometriosis where pelvic endometriosis is also present, local inflammation surrounding the ectopic implants may favor the shedding of endometriotic cells, which can be transported to the umbilicus. Isolated primary umbilical endometriosis is difficult to explain. the disease might arise from metaplastic changes. Various other theories of pathogenesis include hormonal theory, oxidative stress and inflammation theory, immune dysfunction, apoptosis suppression, genetic and stem cells theory. ${ }^{2-4}$

As for clinical presentation, there is a discrete bluishpurple mass in the umbilicus of varying size, becoming swollen, painful and bleeding during menstruation. Some patients can have continuous pain instead of cyclical pain. $^{5}$ In patients with UE the clinical picture and physical examination is the mainstay for diagnosis. Differential diagnosis of umbilical nodules includes cysts, umbilical hernia, hematoma, inflammatory and infectious lesions, and benign and malignant skin neoplasm like granulomas, melanoma and metastatic adenocarcinoma. Computed tomography or MRI can confirm the extent of involvement of underlying structures. CA125 may be raised, secondary to the presence of pelvic lesions. Histological analysis is the gold standard for diagnosis, and immunohistochemistry can be used to resolve diagnostic dilemmas. ${ }^{6,7}$ The risk of malignancy from umbilical endometriosis is reassuringly low. There is one reported case of an umbilical endometrium with malignant transformation patient was noted to have endometriosis coexisting with endometrial adenocarcinoma that had metastasized to the umbilicus, a finding that was noted only at the time of surgery. ${ }^{8}$

Management options should aim at avoiding any risk of recurrence and ruling out co-existent pelvic endometriosis. Fedele et al reviewed treatment in 7 cases of umbilical endometriosis in 2010. In this 7 cases, 2 were primary and rest were secondary. Although its treatment is typically surgical, in literature the approach used is variable and extends from diathermocoagulation to omphalectomy. Such superficial treatments for umbilical endometriosis can predispose the patient to a relapse of the disease. Although a medical treatment can be considered, but it is merely palliative. The treatment of choice in these patients should be that of excisional surgery so as to avoid lesion relapse and the risk of oncogenic transformation with laparoscopy to avoid risk of recurrences and malignant transformation. ${ }^{1,4,9}$ 
Primary umbilical endometriosis because of its rarity has no management guidelines. We recommend a wide local excision of the lesion, with simultaneous explorative laparoscopy to reduce the risk of recurrence and also exclude peritoneal involvement. If there is associated pelvic endometriosis, it needs concurrent management, medical or surgical.

\section{CONCLUSION}

The possibility of endometriosis must be considered during the evaluation of any umbilical mass despite the absence of previous surgery, paying special attention to menstrual symptoms or bloody discharge. Complete excision with the help of laparoscopic evaluation and successive histology are highly recommended for obtaining a definitive diagnosis and optimal treatment.

\section{Funding: No funding sources}

Conflict of interest: None declared

Ethical approval: Not required

\section{REFERENCES}

1. Fancellu A, Pinna A, Manca A, Capobianco G, Porcua A. Primary umbilical endometriosis. Case report and discussion on management options. Int $\mathbf{J}$ Surg Case Rep. 2013;4(12):1145-8.
2. Mizutani T, Sakamoto Y, Ochiai H, Maeshima A. umbilical endometriosis with urachal remnant. Arch Dermatol. 2012;148(11):1331-2.

3. Calagna G, Perino A, Chianetta D, Vinti D, Triolo MM, Rimi C, et al. Primary umbilical endometrioma: analyzing the pathogenesis of endometriosis from an unusual localization. Taiwanese Journal of Obstetrics and Gynecology. 2015;54(3):306-12.

4. Pallavi VB, Giurguis MM. Menstruating from the umbilicus as a rare case of primary umbilical endometriosis: a case report. J Med Case Rep. 2009;3:9326.

5. Efremidou EI, Kouklakis G, Mitrakas A, Liratzopoulos N, Polychronidis ACH. Primary umblical endometrioma: a rare cause of spontaneous abdominal wall endometriosis. Int $\mathrm{J}$ Gen Med. 2012;5:999-1002.

6. Victory R, Diamond MP, Johns DA. Villar's nodule: a case report and systematic literature review of endometriosis external of the umbilicus. J Minim Invasive Gynecol. 2007;14:23-32.

7. Obata K, Ikoma N, Oomura G, Inoue Y. Clear cell adenocarcinoma arising from umbilical endometriosis. Journal of Obstetrics and Gynaecology Research. 2013;39:455-61.

8. Fedele L, Frontino G, Bianchi S, Borruto F, Ciappina $\mathrm{N}$. Umbilical endometriosis: a radical excision with laparoscopic assistance. Int J Surg. 2010;8(2):109-11.

Cite this article as: Malik R, Sharma $\mathrm{P}$. Menstruating umbilicus! A rare case report of primary umbilical endometriosis with review of literature. Int J Reprod Contracept Obstet Gynecol 2016;5:2453-6. 